\title{
GLOBAL DIMENSION OF DIFFERENTIAL OPERATOR RINGS. II
}

\author{
BY
}

K. R. GOODEARL

\begin{abstract}
The aim of this paper is to find the global homological dimension of the ring of linear differential operators $R\left[\theta_{1}, \ldots, \theta_{u}\right]$ over a differential ring $R$ with $u$ commuting derivations. When $R$ is a commutative noetherian ring with finite global dimension, the main theorem of this paper (Theorem 21) shows that the global dimension of $R\left[\theta_{1}, \ldots, \theta_{u}\right]$ is the maximum of $k$ and $q+u$, where $q$ is the supremum of the ranks of all maximal ideals $M$ of $R$ for which $R / M$ has positive characteristic, and $k$ is the supremum of the sums $\operatorname{rank}(P)+\operatorname{diff} \operatorname{dim}(P)$ for all prime ideals $P$ of $R$ such that $R / P$ has characteristic zero. [The value $\operatorname{diff} \operatorname{dim}(P)$ is an invariant measuring the differentiability of $P$ in a manner defined in §3.] In case we are considering only a single derivation on $R$, this theorem leads to the result that the global dimension of $R[\theta]$ is the supremum of $\operatorname{gl} \operatorname{dim}(R)$ together with one plus the projective dimensions of the modules $R / J$, where $J$ is any primary differential ideal of $R$. One application of these results derives the global dimension of the Weyl algebra in any degree over any commutative noetherian ring with finite global dimension.
\end{abstract}

1. Introduction. As in [5], we reserve the term differential ring for a nonzero associative ring $R$ with unit together with a single specified derivation $\delta$ on $R$. In case we have specified a finite collection $\delta_{1}, \ldots, \delta_{u}$ of commuting derivations on $R$, we shall refer to $R$ as a $u$-differential ring. The ring of differential operators over a $u$-differential ring $R$ is additively the group of all polynomials over $R$ in indeterminates $\theta_{1}, \ldots, \theta_{u}$, with multiplication subject to the requirements $\theta_{i} \theta_{j}=\theta_{j} \theta_{i}$ for all $i, j$, and $\theta_{i} a=a \theta_{i}+\delta_{i} a$ for all $i$, all $a \in R$. We denote this ring by $R\left[\theta_{1}, \ldots, \theta_{u}\right]$, or by $R[\theta]$ in the case of a single derivation. The elements of $R\left[\theta_{1}, \ldots, \theta_{u}\right]$ are normally written as sums of monomials of the form $r p$, where $r \in R$ and $p$ is a product of powers of the $\theta_{i}$, although for some arguments it is more convenient to use right-hand coefficients. (Note that when an element of $R\left[\theta_{1}, \ldots, \theta_{u}\right]$ is written with left-hand coefficients, these coefficients will in general be different from those used to express

Presented to the Society, January 18, 1974 under the title Homological dimension of differential operator rings; received by the editors October 1, 1973.

AMS (MOS) subject classifications (1970). Primary 16A60; Secondary $12 \mathrm{H05}$.

Key words and phrases. Global dimension, rings of linear differential operators, differential algebra, Weyl algebras. 
the element with right-hand coefficients.) In particular, any element $x \in R[\theta]$ is written as $x=r_{0}+r_{1} \theta+\ldots+r_{n} \theta^{n}$ for suitable $r_{i} \in R$, and when $r_{n} \neq 0$ we say that $n$ is the degree of $x$ and that $r_{n}$ is the leading coefficient of $x$. Finally, for induction purposes we note that

$$
R\left[\theta_{1}, \ldots, \theta_{u}\right]=R\left[\theta_{1}, \ldots, \theta_{u-1}\right]\left[\theta_{u}\right],
$$

where $\delta_{u}$ has been implicitly extended to $R\left[\theta_{1}, \ldots, \theta_{u-1}\right]$ by setting $\delta_{u} \theta_{i}=0$ for all $i$.

The objective of this paper is to derive formulas for the global dimension of $R\left[\theta_{1}, \ldots, \theta_{u}\right]$, where $R$ is a commutative noetherian $u$-differential ring with finite global dimension. Basically, the task breaks down into the problems of finding suitable lower bounds and upper bounds for the global dimension of $R\left[\theta_{1}, \ldots, \theta_{u}\right]$. Since these two problems require relatively different techniques, we allot separate sections of the paper to them. In both cases we also require the techniques of localization: namely ordinary localization of the commutative ring $R$ at a prime ideal, which induces a natural noncommutative localization on the ring $R\left[\theta_{1}, \ldots, \theta_{u}\right]$.

Our notation for the various homological dimensions involved with a ring $S$ is as follows: $\mathrm{r}$ gl dim $S$ denotes the right global dimension of $S$, and $\operatorname{GWD}(S)$ denotes the global weak dimension of $S$. For any $S$-module $A$, we use $\operatorname{pd}_{S}(A)$ and $\operatorname{wd}_{S}(A)$ to stand for the respective projective and weak dimensions of $A$. The reason that weak dimensions are useful is that we shall be dealing mostly with noetherian rings. For if $R$ is a right and left noetherian differential ring, then $R[\theta]$ is right and left noetherian, as observed in [2, p. 68]. By induction, $R\left[\theta_{1}, \ldots, \theta_{u}\right]$ is right and left noetherian also. Our basic estimates on homological dimensions are given in the following two propositions, which follow automatically by induction from [5, Propositions 2,3$]$.

Proposition 1. Let $R$ be any $u$-differential ring, and set $T=R\left[\theta_{1}, \ldots, \theta_{u}\right]$. If $A$ is any right $T$-module, then

$$
\mathrm{pd}_{R}(A) \leqslant \mathrm{pd}_{T}(A) \leqslant u+\mathrm{pd}_{R}(A) .
$$

PROPOSITION 2. If $R$ is any $u$-differential ring with $\mathrm{r} \operatorname{gl} \operatorname{dim} R<\infty$, then $\mathrm{r} \mathrm{gl} \operatorname{dim} R \leqslant \mathrm{r} g l \operatorname{dim} R\left[\theta_{1}, \ldots, \theta_{u}\right] \leqslant u+\mathrm{r} g l \operatorname{dim} R$.

The left-hand inequality in Proposition 2 may fail if $\mathrm{r} g l \operatorname{dim} R=\infty$, as shown in $[5, \S 2]$.

We close this section with two propositions which give the basic results on the localization procedures needed later. The first of these is proved in exactly the same manner as [5, Lemma 7]. 
Proposition 3. Let $R$ be any commutative $u$-differential ring, and set $T=R\left[\theta_{1}, \ldots, \theta_{u}\right]$. If $S$ is any multiplicatively closed subset of $R$, then the following are true:

(a) Each $\delta_{i}$ induces a derivation on $R_{S}$ according to the rule $\delta_{i}(r / s)=$ $\left[\left(\delta_{i} r\right) s-r\left(\delta_{i} s\right)\right] / s^{2}$.

(b) The natural map $T \rightarrow R_{S}\left[\theta_{1}, \ldots, \theta_{u}\right]$ makes $R_{S}\left[\theta_{1}, \ldots, \theta_{u}\right]$ into a flat right and left $T$-module such that the multiplication map $R_{S}\left[\theta_{1}, \ldots, \theta_{u}\right] \otimes_{T} R_{S}\left[\theta_{1}, \ldots, \theta_{u}\right] \rightarrow R_{S}\left[\theta_{1}, \ldots, \theta_{u}\right]$ is an isomorphism.

(c) $\mathrm{r} g l \operatorname{dim} R_{S}\left[\theta_{1}, \ldots, \theta_{u}\right] \leqslant \mathrm{r} g l \operatorname{dim} T$.

PROPOSITION 4. Let $R$ be a commutative noetherian u-differential ring with $\mathrm{gl} \operatorname{dim} R<\infty$. Then

$\mathrm{r} \mathrm{gl} \operatorname{dim} R\left[\theta_{1}, \ldots, \theta_{u}\right]$

$$
=\sup \left\{\mathrm{r} g l \operatorname{dim} R_{M}\left[\theta_{1}, \ldots, \theta_{u}\right] \mid M \text { is a maximal ideal of } R\right\} \text {. }
$$

Proof. Inasmuch as all rings involved in this proposition are right noetherian, it suffices to prove the corresponding statement for global weak dimension. Just as in the proof of [5, Lemma 7], we see that each of the rings $R_{M}\left[\theta_{1}, \ldots, \theta_{u}\right]$ is a classical localization of $R\left[\theta_{1}, \ldots, \theta_{u}\right]$ with respect to the multiplicative set $R \backslash M$. It is easily checked that these localizations satisfy the hypotheses of [13, Proposition 1], from which we obtain the desired result.

2. Lower bounds. In this section we set up our basic tool for finding lower bounds for the global dimension of $R\left[\theta_{1}, \ldots, \theta_{u}\right]$. This is Theorem 7, which allows us to compute the projective dimensions of those $R\left[\theta_{1}, \ldots, \theta_{u}\right]$ modules which happen to be finitely generated as $R$-modules. As one consequence, we find that $\mathrm{r} g \mathrm{dim} R\left[\theta_{1}, \ldots, \theta_{u}\right] \geqslant u+\operatorname{rank}(M)$ for any maximal ideal $M$ of $R$ such that $R / M$ has positive characteristic. We begin with two lemmas, the first of which is essentially a special case of [6, Lemma, p. 68]

LEMma 5. Let $R$ be any differential ring, and let $A$ be a right $R[\theta]$-module. If $E: 0 \rightarrow K \rightarrow F \rightarrow A \rightarrow 0$ is an exact sequence of right $R$-modules with $F_{R}$ free, then $K$ and $F$ can be made into right $R[\theta]$-modules such that $E$ becomes an exact sequence of $R[\theta]$-modules.

Proof. Let $f: K \rightarrow F$ and $g: F \rightarrow A$ denote the maps in $E$. Choosing a decomposition of $F$ as a direct sum of copies of $R$, and applying $\delta$ to each copy of $R$, we obtain an additive map $d: F \rightarrow F$ such that $d(x r)=(d x) r+$ $x(\delta r)$ for all $x \in F, r \in R$. Define a map $h: F \rightarrow A$ by the rule $h x=g(d x)+$ $(g x) \theta$, and check that $h$ is an $R$-homomorphism. Then $h$ lifts to an $R$-homomorphism $k: F \rightarrow F$ such that $g k=h$. 
Now $d^{\prime}=k-d$ is an additive endomorphism of $F$ such that $d^{\prime}(x r)=$ $\left(d^{\prime} x\right) r-x(\delta r)$ for all $x \in F, r \in R$, from which we infer that $F$ can be made into a right $R[\theta]$-module by defining $x \theta=d^{\prime} x$ for all $x \in F$. Computing that now $g(x \theta)=(g x) \theta$ for all $x \in F$, we see that $g$ is an $R[\theta]$-homomorphism. As a consequence, $\operatorname{ker} g$ is an $R[\theta]$-submodule of $F$, hence $K$ can be made into a right $R[\theta]$-module so that $f$ is an $R[\theta]$-homomorphism.

LEMma 6. Let $R$ be a semiprime left Goldie differential ring. If $J$ is any essential left ideal of $R[\theta]$, then $J$ contains an element of $R[\theta]$ whose leading coefficient is a regular element of $R$.

Proof. Since $R$ is left Goldie, it must contain a finite direct sum $A_{1} \oplus$ $\ldots \oplus A_{k}$ of nonzero uniform left ideals which is essential in ${ }_{R} R$. The essentiality of $J$ implies that each of the left ideals $R[\theta] A_{i}$ must contain a nonzero element $x_{i}$ from $J$. After multiplying the $x_{i}$ on the left by suitable powers of $\theta$, we may assume that the $x_{i}$ all have the same degree, say $n$. Inasmuch as $R[\theta]=R+\theta R+\theta^{2} R+\ldots$, we see that $R[\theta] A_{i}=A_{i}+\theta A_{i}+\theta^{2} A_{i}+\ldots$ Noting that the degree of $x_{i}$ remains the same when $x_{i}$ is written with coefficients on the right, we see that $x_{i}=x_{i 0}+\theta x_{i 1}+\ldots+\theta^{n-1} x_{i, n-1}+\theta^{n} a_{i}$ for some $x_{i j}, a_{i} \in A_{i}, a_{i} \neq 0$. Changing back to left-hand coefficients, the leading coefficient of $x_{i}$ is still $a_{i}$, although the other coefficients need not even belong to $A_{i}$.

Now $R a_{i}$ is a nonzero submodule of the uniform left ideal $A_{i}$ and hence is essential in $A_{i}$, from which we deduce that $R a_{1} \oplus \ldots \oplus R a_{k}$ is an essential left ideal of $R$. Inasmuch as $R$ is a semiprime left Goldie ring, [8, Lemma 7.2.5] says that $R a_{1} \oplus \ldots \oplus R a_{k}$ must contain a regular element $a$ of $R$, say $a=$ $r_{1} a_{1}+\ldots+r_{k} a_{k}$. Since each $x_{i}$ has leading term $a_{i} \theta^{n}$, we now conclude that $r_{1} x_{1}+\ldots+r_{k} x_{k}$ is an element of $J$ whose leading coefficient is $a$.

THEOREM 7. Let $R$ be a semiprime right and left noetherian $u$-differential ring, and set $T=R\left[\theta_{1}, \ldots, \theta_{u}\right]$. If $A$ is any nonzero right $T$-module such that $A_{R}$ is finitely generated, then $\operatorname{pd}_{T}(A)=u+\operatorname{pd}_{R}(A)$.

Proof. Each of the rings $T_{j}=R\left[\theta_{1}, \ldots, \theta_{j}\right]$ is right and left noetherian, and it is easily checked that each $T_{j}$ is semiprime as well. Now $A$ is a finitely generated right $T_{j}$-module for each $j$, and we are done if we show that the projective dimension of $A$ over each $T_{j+1}$ is exactly one greater than the projective dimension of $A$ over $T_{j}$. Thus it suffices to consider only the 1-differential case: here $R$ is a semiprime right and left noetherian differential ring, $A$ is a nonzero right $R[\theta]$-module such that $A_{R}$ is finitely generated, and we must prove that $\operatorname{pd}_{R[\theta]}(A)=1+\operatorname{pd}_{R}(A)$. 
The case $\operatorname{pd}_{R}(A)=\infty$ is taken care of by Proposition 1, hence we may assume that $\operatorname{pd}_{R}(A)=n<\infty$, and we induct on $n$. As noted above, $R[\theta]$ is a semiprime right and left noetherian ring, hence the maximal right quotient ring $Q$ of $R[\theta]$ coincides with the maximal left quotient ring of $R[\theta]$ (and is a classical right and left quotient ring). Also, $R[\theta]$ is a semiprime right Goldie ring, hence [14, Theorem 1.7] shows that $R[\theta]$ is a right nonsingular ring.

If $n=0$, then $\operatorname{pd}_{R[\theta]}(A) \leqslant 1$ by Proposition 1 ; hence it remains to show that $A_{R[\theta]}$ is not projective. Inasmuch as $A \neq 0$ and all projective right $R[\theta]$-modules are nonsingular, it suffices to show that $A_{R[\theta]}$ is singular. Given any $a \in A$, set $J=\{x \in R[\theta] \mid a x=0\}$ and note that $R[\theta] / J$ is noetherian as an $R$-module. Now any nonzero right ideal $K$ of $R[\theta]$ contains elements of arbitrarily high degree, whence $K_{R}$ cannot be finitely generated. Thus the natural map $K \rightarrow R[\theta] \rightarrow R[\theta] / J$ cannot be a monomorphism, i.e., $K \cap J \neq 0$. Therefore $J$ is an essential right ideal of $R[\theta]$ and so $A$ is indeed a singular $R[\theta]$-module.

Next assume that $n=1$, and choose a positive integer $k$ such that $A_{R}$ can be generated by $k$ elements. If $S$ denotes the ring of all $k \times k$ matrices over $R$, then we obtain a Morita equivalence between the category of all right $R$-modules and the category of all right $S$-modules, where any right $R$-module $B$ gets taken to $B \otimes_{R} R^{k}$, i.e., to $B^{k}$. We intend to use this equivalence to transfer our problem to $S$-modules, since $A^{k}$ is a cyclic right $S$-module. Now $\delta$ can be extended to a derivation of $S$ by letting $\delta$ act on each entry of any matrix in $S$, and then $S[\theta]$ may be identified with the ring of all $k \times k$ matrices over $R[\theta]$. With this identification, we get another Morita equivalence between the category of all right $R[\theta]$-modules and the category of all right $S[\theta]$-modules, where any right $R[\theta]$-module $B$ gets taken to $B^{k}$. Because of these equivalences, $\operatorname{pd}_{S}\left(A^{k}\right)=1$ and $\operatorname{pd}_{R[\theta]}(A)=\operatorname{pd}_{S[\theta]}\left(A^{k}\right)$, hence we may assume without loss of generality that $A_{R}$ is cyclic.

Therefore we may assume that $A=R / I$ for some right ideal $I$ of $R$. Inasmuch as $A$ is also a right $R[\theta]$-module, we have $\overline{1} \theta=\bar{\alpha}$ for some $\alpha \in R$. Then $\bar{r} \theta=\overline{(\alpha-\delta) r}$ for all $r \in R$ and consequently $(\alpha-\delta)(I) \subseteq I$. Noting that $R[\theta]=$ $R+(\theta-\alpha) R[\theta]$, we see that $A \cong R[\theta] / J$, where $J=I+(\theta-\alpha) R[\theta]$.

We claim that for any $R[\theta]$-homomorphism $f: J \rightarrow R[\theta], f l_{I}$ must be left multiplication by some element of $R[\theta]$. Since $R[\theta]$ is a right nonsingular ring, its maximal right quotient ring $Q$ is the injective hull of $R[\theta]_{R[\theta]}$, hence $f$ must be left multiplication by some $t \in Q$. Noting that $t(\theta-\alpha) \in$ $R[\theta]$, we see that $t=x(\theta-\alpha)^{-1}$ for some $x \in R[\theta]$. This element $x$ can be put in the form $x=x_{0}+x_{1}(\theta-\alpha)$ for suitable $x_{0} \in R$ and $x_{1} \in R[\theta]$, whence $t=x_{0}(\theta-\alpha)^{-1}+x_{1}$. If $x_{0}=0$, then $f$ itself is left multiplication by the 
element $x_{1} \in R[\theta]$ and the claim holds, hence we may assume that $x_{0} \neq 0$. We have $t J=f J \subseteq R[\theta]$, and clearly $x_{1} J \subseteq R[\theta]$ as well, whence $x_{0}(\theta-\alpha)^{-1} J$ $\subseteq R[\theta]$.

Inasmuch as $Q$ is also the maximal left quotient ring of $R[\theta]$, we must have $K x_{0}(\theta-\alpha)^{-1} \subseteq R[\theta]$ for some essential left ideal $K$ of $R[\theta]$, and by Lemma $6, K$ must contain an element $y$ whose leading coefficient is a regular element of $R$. Now $y$ is clearly a regular element of $R[\theta]$ and so is invertible in $Q$, hence we obtain $x_{0}(\theta-\alpha)^{-1}=y^{-1} z$ for some $z \in R[\theta]$, or $y x_{0}=$ $z(\theta-\alpha)$. Since $x_{0} \neq 0$ we have $z \neq 0$, too, which makes it possible to talk about the degrees of the elements in this last equation. Obviously $\operatorname{deg}[z(\theta-\alpha)]$ $=1+\operatorname{deg}(z)$, and since the leading coefficient of $y$ is a regular element we obtain $\operatorname{deg}\left(y x_{0}\right)=\operatorname{deg}(y)$; thus $\operatorname{deg}(y)=1+\operatorname{deg}(z)$. Given any $r \in I$, we have $y^{-1} z r=x_{0}(\theta-\alpha)^{-1} r \in R[\theta]$ (because $r \in J$ ), whence $z r \in y R[\theta]$. Since $\operatorname{deg}(y)>\operatorname{deg}(z)$, and since $\operatorname{deg}(y w) \geqslant \operatorname{deg}(y)$ for all nonzero $w \in R[\theta]$, this is possible only when $z r=0$. Thus we obtain $z I=0$, from which we infer that $x_{0}(\theta-\alpha)^{-1} I=0$. It follows that $\left.f\right|_{I}$ is just left multiplication by the element $x_{1} \in R[\theta]$, as claimed.

As right $R$-modules, $J=I \oplus(\theta-\alpha) R[\theta]$, from which we see that $I$ can be made into a right $R[\theta]$-module so that the projection $p: J \rightarrow I$ is an $R[\theta]$-homomorphism. Choose an $R[\theta]$-epimorphism $g: F \rightarrow I$, where $F$ is a finitely generated free right $R[\theta]$-module. If we assume that $J_{R[\theta]}$ is projective, then $p$ must lift to an $R[\theta]$-homomorphism $h: J \rightarrow F$ such that $g h=p$. In view of the claim just proved above, we see that $\left.h\right|_{I}$ must be left multiplication by some $w \in F$, from which we compute that $(g w) r=r$ for all $r \in I$. Consequently $g w$ is an idempotent and $(g w) R=I$, hence $\left(R / I_{R}\right.$ must be projective. However, this contradicts the assumption that $\operatorname{pd}_{R}(A)=1$, and thus $J_{R[\theta]}$ cannot be projective. This gives us $\mathrm{pd}_{R[\theta]}(A)>1$, so by Proposition 1 we conclude that $\operatorname{pd}_{R[\theta]}(A)=2$.

Finally, let $n>1$ and assume the theorem holds for $n-1$. Choose an exact sequence $E: 0 \rightarrow K \rightarrow F \rightarrow A \rightarrow 0$ of right $R$-modules with $F_{R}$ finitely generated free, and use Lemma 5 to make $E$ into an exact sequence of right $R[\theta]$-modules. Now $K$ is a right $R[\theta]$-module which is finitely generated as an $R$-module, and $\operatorname{pd}_{R}(K)=n-1>0$ (so that in particular $K \neq 0$ ), hence we obtain $\operatorname{pd}_{R[\theta]}(K)=n$ from the induction hypothesis. Inasmuch as $n>\cdot 1$ and $\operatorname{pd}_{R[\theta]}(F) \leqslant 1$ by Proposition 1 , it now follows from the long exact sequence for Ext that $\operatorname{pd}_{R[\theta]}(A)=n+1$.

Using more homological methods, a stronger version of Theorem 7 has been proved in [12, Corollary $1.7(\mathrm{~b})]$. 
I am grateful to the referee for pointing out the necessity of condition (b) in the following corollary.

COROLlaRY 8. Let $R$ be a semiprime right and left noetherian $u$-differential ring, let $J$ be a proper right ideal of $R$, and let $\alpha_{1}, \ldots, \alpha_{u} \in R$ such that

(a) $\left(\delta_{i}-\alpha_{i}\right)(J) \subseteq J$ for $i=1, \ldots, u$,

(b) $\left(\delta_{i}-\alpha_{i}\right)\left(\alpha_{j}\right)-\left(\delta_{j}-\alpha_{j}\right)\left(\alpha_{i}\right) \in J$ for $i, j=1, \ldots, u$. Then $\mathrm{r} g l \operatorname{dim} R\left[\theta_{1}, \ldots, \theta_{u}\right] \geqslant u+\mathrm{pd}_{R}(R / J)$.

Proof. Let $A=R / J$, which is a nonzero finitely generated right $R$-module. Using (a) and (b), we infer that $A$ can be made into a right $R\left[\theta_{1}, \ldots, \theta_{u}\right]$ module by setting $\bar{r} \theta_{i}=\overline{\left(\alpha_{i}-\delta_{i}\right) r}$ for all $i$ and all $r \in R$. (Condition (a) ensures that $x \theta_{i}$ is well defined, and condition (b) ensures that $x \theta_{i} \theta_{j}=x \theta_{j} \theta_{i}$. The details are very straightforward.) Consequently, Theorem 7 says that $A$ is a right $R\left[\theta_{1}, \ldots, \theta_{u}\right]$-module with projective dimension $u+\operatorname{pd}_{R}(A)$.

Corollary 8 applies in particular to the case when $J$ is a differential right ideal of $R$, i.e., $\delta_{i}(J) \subseteq J$ for all $i$. In this case, condition (b) is trivially satisfied.

In order to apply Theorem 7 or Corollary 8 in the case when $R$ is a commutative noetherian ring of finite global dimension, we must know that $R$ is semiprime. This is probably well known, as are the other facts in the following proposition, which we include for completeness.

PROPOSITION 9. Let $R$ be any commutative noetherian ring with gl $\operatorname{dim} R=n<\infty$.

(a) $R$ is a finite direct product of integral domains, and thus is a semiprime ring.

(b) If $M$ is any maximal ideal of $R$, then $\mathrm{gl} \operatorname{dim} R_{M}=\operatorname{rank}(M)=$ $\operatorname{pd}_{R}(R / M) \leqslant n$.

(c) The (classical) Krull dimension of $R$ is $n$.

Proof. (a) For each maximal ideal $M$ of $R$ [9, Part III, Theorem 11] says that $\mathrm{gl} \operatorname{dim} R_{M} \leqslant n<\infty$, hence it follows from [9, Part III, Theorem 13] that $R_{M}$ is a regular local ring. Thus $R_{M}$ is an integral domain for every maximal ideal $M$ [10, Theorem 164], whence [10, Theorem 168] says that $R$ is a finite direct product of integral domains.

(b) As seen in (a), $R_{M}$ is a regular local ring. According to [9, Part III, Theorem 12], gl $\operatorname{dim} R_{M}$ is the same as the Krull dimension of $R_{M}$, i.e., $\mathrm{gl} \operatorname{dim} R_{M}=\operatorname{rank}(M)$. In view of [10, Theorem 176], we also see that the projective dimension of $R_{M} / M R_{M}$ over $R_{M}$ is equal to $\operatorname{rank}(M)$. Inasmuch as 
$R$ is noetherian, the projective dimension of any finitely generated $R$-module $A$ is the supremum of the projective dimensions of the $R_{K}$-modules $A_{K}$, where $K$ ranges over all maximal ideals of $R$. For the case $A=R / M$, we have $A_{M}=$ $R_{M} / M R_{M}$ and $A_{K}=0$ for all other $K$, from which we conclude that $\mathrm{pd}_{R}(R / M)$ $=\operatorname{rank}(M)$.

(c) Since $R$ is noetherian, $n$ is the supremum of the numbers $\mathrm{gl} \operatorname{dim} R_{M}$ over all maximal ideals $M$, hence (c) follows immediately from (b).

We conclude this section by deriving the lower bound $u+\operatorname{rank}(M) \leqslant$ $\mathrm{r} g l \operatorname{dim} R\left[\theta_{1}, \ldots, \theta_{u}\right]$, where $M$ is any maximal ideal of $R$ such that $R / M$ has positive characteristic. We must also derive lower bounds for $\mathrm{r} g \mathrm{dim} R\left[\theta_{1}, \ldots, \theta_{u}\right]$ related to maximal ideals $M$ such that $R / M$ has characteristic zero, but this depends on the differential dimension of $M$, which we develop in the next section.

PROPOSITION 10. Let $R$ be a commutative noetherian u-differential ring with $\mathrm{gl} \operatorname{dim} R<\infty$, and let $M$ be a maximal ideal of $R$. If $R / M$ has characteristic $p>0$, then

$\mathrm{r} g \mathrm{dim} R\left[\theta_{1}, \ldots, \theta_{u}\right] \geqslant u+\operatorname{rank}(M)$.

Proof. According to Proposition 9, the simple module $R / M$ satisfies the property $\operatorname{pd}_{R}(R / M)=\operatorname{rank}(M)<\infty$. If $A$ is any nonzero $R$-module with a composition series such that all the composition factors are isomorphic to $R / M$, then it follows from the long exact sequence for Ext (by induction on length) that $\operatorname{pd}_{R}(A)=\operatorname{rank}(M)$.

Now let $J$ be the ideal of $R$ generated by $p R$ and $\left\{x^{p} \mid x \in M\right\}$, and note that $\delta_{i}(J) \subseteq J$ for all $i=1, \ldots, u$. Since $\operatorname{char}(R / M)=p$, we see that $J \subseteq M$, whence $R / J \neq 0$. Inasmuch as $M / J$ is a nil ideal in the noetherian ring $R / J$, Levitzki's Theorem says that $M / J$ must be nilpotent, from which we infer that $R / J$ has a composition series with all composition factors isomorphic to $R / M$. Now $\operatorname{pd}_{R}(R / J)=\operatorname{rank}(M)$, hence the desired inequality follows from Corollary 8.

COROLLARY 11. Let $R$ be a commutative noetherian u-differential ring with $\mathrm{gl} \operatorname{dim} R=n<\infty$. If $R$ has positive characteristic, then $\mathrm{r} \mathrm{gl} \operatorname{dim} R\left[\theta_{1}, \ldots, \theta_{u}\right]=n+u$.

Proof. In view of Proposition 9, we must have $\operatorname{rank}(M)=n$ for some maximal ideal $M$, whence Proposition 10 yields $\mathrm{r} g l \operatorname{dim} R\left[\theta_{1}, \ldots, \theta_{u}\right] \geqslant$ $n+u$. According to Proposition 1 , we also have $\mathrm{r} g l \operatorname{dim} R\left[\theta_{1}, \ldots, \theta_{u}\right] \leqslant$ $n+u$. 
3. Differential dimension. The purpose of this section is to introduce a concept of differential dimension for prime ideals $P$ of $R$, and to obtain the lower bounds

$$
\operatorname{rank}(P)+\operatorname{diff} \operatorname{dim}(P) \leqslant \mathrm{r} g l \operatorname{dim} R\left[\theta_{1}, \ldots, \theta_{u}\right] .
$$

This differential dimension of $P$ is meant to measure the "differentiability" of $P$ in the sense that it indicates how large a collection of $R$-linear combinations of the derivations $\delta_{1}, \ldots, \delta_{u}$ can map $P$ into itself. In particular, the differential dimension of $P$ will be $u$ if and only if $P$ is closed under all the $\delta_{i}$. The details follow.

Given any commutative $u$-differential ring $R$, make $\operatorname{Hom}_{Z}(R, R)$ into a left $R$-module by defining $(r f)(x)=r(f x)$ for all $r, x \in R, f \in \operatorname{Hom}_{Z}(R, R)$, and let $\Delta$ denote the left $R$-submodule of $\operatorname{Hom}_{Z}(R, R)$ generated by $\delta_{1}, \ldots$, $\delta_{u}$. For any prime ideal $P$ of $R$, the set $D(P)=\{f \in \Delta \mid f(P) \subseteq P\}$ is a left $R$-submodule of $\Delta$, and it is clear that $\Delta / D(P)$, is a torsion-free left $(R / P)$-module. We define the differential codimension of $P$, abbreviated $\operatorname{diff} \operatorname{codim}(P)$, to be the rank of this torsion-free $(R / P)$-module $\Delta / D(P)$, i.e., the vector space dimension $[Q[\Delta / D(P)]: Q]$, where $Q$ stands for the quotient field of $R / P$. [Alternately, diff $\operatorname{codim}(P)$ may be defined as the Goldie dimension of the left $R$-module $\Delta / D(P)$.] Finally, we define the differential dimension of $P$, denoted $\operatorname{diff} \operatorname{dim}(P)$, to be $u$ - diff $\operatorname{codim}(P)$.

Proposition 12. Let $R$ be a commutative u-differential ring. Let $P$ be any prime ideal of $R$, and set $S=R_{P}, M=P R_{P}$. Then each $\delta_{i}$ induces a linear transformation $\delta_{i}^{*}$ in the dual space $V=\operatorname{Hom}_{S / M}\left(M / M^{2}, S / M\right)$, and the subspace $W$ of $V$ spanned by $\delta_{1}^{*}, \ldots, \delta_{u}^{*}$ has dimension exactly $\operatorname{diff} \operatorname{codim}(P)$.

Proof. Each $\delta_{i}$ induces a derivation on $S$ as in Proposition 3, and this gives us additive maps $\delta_{i}: M \rightarrow S$. Observing that $\delta_{i}\left(M^{2}\right) \subseteq M$, we see that $\delta_{i}$ induces an additive map $\delta_{i}^{*}: M / M^{2} \rightarrow S / M$, and an easy check confirms that $\delta_{i}^{*}$ is an $(S / M)$-homomorphism.

There is a left $R$-homomorphism $\phi: \Delta \rightarrow W$ such that $\phi\left(\delta_{i}\right)=\delta_{i}^{*}$ for each $i$, and an easy computation shows that $\operatorname{ker} \phi=D(P)$. Now $\phi \Delta$ is a left module over the domain $T=(R+M) / M \cong R / P$, from which we infer that ${ }_{T}(\phi \Delta)$ and ${ }_{R / P}[\Delta / D(P)]$ have the same rank, i.e., ${ }_{T}(\phi \Delta)$ has rank $\operatorname{diff} \operatorname{codim}(P)$. Inasmuch as ${ }_{T}(\phi \Delta)$ is torsion-free and $S / M$ is the quotient field of $T$, the rank of ${ }_{T}(\phi \Delta)$ is just $[S(\phi \Delta): S / M]$. Observing that $S(\phi \Delta)=W$, we conclude that $[W: S / M]=$ $\operatorname{diff} \operatorname{codim}(P)$.

COROllary 13. Let $R$ be a commutative u-differential ring. If $P \subseteq Q$ are prime ideals of $R$, then $\operatorname{diff} \operatorname{codim}\left(P R_{Q}\right)=\operatorname{diff} \operatorname{codim}(P)$. 
Proof. Inasmuch as the localization of $R_{Q}$ at the prime ideal $P R_{Q}$ is just $R_{P}$, this follows immediately from Proposition 12 .

In particular, Corollary 13 shows that $\operatorname{diff} \operatorname{codim}(P)=\operatorname{diff} \operatorname{codim}\left(P R_{P}\right)$ for any prime ideal $P$, which makes it possible to carry out some computations using the maximal ideal $P R_{P}$ in the local ring $R_{P}$. Before proving the inequality $\operatorname{rank}(P)+\operatorname{diff} \operatorname{dim}(P) \leqslant \mathrm{r} g l \operatorname{dim} R\left[\theta_{1}, \ldots, \theta_{u}\right]$, we introduce the following easy lemma, which will also be useful later.

LEMMA 14. (a) Let $R$ be any ring such that $\mathrm{r} g l \operatorname{dim} R=n<\infty$. If $A \subseteq B$ are right $R$-modules with $\mathrm{pd}_{R}(A)=n$, then $\operatorname{pd}_{R}(B)=n$.

(b) Let $R$ be any ring such that $\mathrm{GWD}(R)=n<\infty$. If $A \subseteq B$ are $R$-modules with $\operatorname{wd}_{R}(A)=n$, then $\operatorname{wd}_{R}(B)=n$.

Proof. (a) If $\operatorname{pd}_{R}(B)<n$, then it follows from the long exact sequence for Ext that $\operatorname{pd}_{R}(B / A)=n+1$, which is impossible. (b) is proved similarly.

PROPOSITION 15. Let $R$ be a commutative noetherian u-differential ring with $\mathrm{gl} \operatorname{dim} R<\infty$. If $P$ is any prime ideal of $R$, then

$$
\mathrm{r} g l \operatorname{dim} R\left[\theta_{1}, \ldots, \theta_{\underline{u}}\right] \geqslant \operatorname{rank}(P)+\operatorname{diff} \operatorname{dim}(P) .
$$

Proof. The local ring $R_{P}$ is a commutative noetherian $u$-differential ring with $\mathrm{gl} \operatorname{dim} R_{P}<\infty$ and certainly $\operatorname{rank}\left(P R_{P}\right)=\operatorname{rank}(P)$. Inasmuch as $\operatorname{diff} \operatorname{dim}\left(P R_{P}\right)=\operatorname{diff} \operatorname{dim}(P)$ by Corollary 13 and $\mathrm{r} g l \operatorname{dim} R_{P}\left[\theta_{1}, \ldots, \theta_{u}\right] \leqslant$ $\mathrm{r} g \mathrm{~d} \operatorname{dim} R\left[\theta_{1}, \ldots, \theta_{u}\right]$ by Proposition 3 , it suffices to consider the case when $R$ is local and $P$ is its maximal ideal. According to Proposition 9, we have gl $\operatorname{dim} R=\operatorname{rank}(P)=\operatorname{pd}_{R}(R / P)$; let $n$ denote this common value.

If $s=\operatorname{diff} \operatorname{codim}(P)$, then Proposition 12 shows that the subspace $W$ of $\operatorname{Hom}_{R / P}\left(P / P^{2}, R / P\right)$ spanned by the induced linear transformations $\delta_{1}^{*}, \ldots, \delta_{u}^{*}$ has dimension $s$. Thus $W$ must have a basis consisting of $s$ of the $\delta_{i}^{*}$, hence we may arrange the indices $1, \ldots, u$ so that $\delta_{1}^{*}, \ldots, \delta_{s}^{*}$ is a basis for $W$.

Since $R$ is semiprime by Proposition 9, the ring $Q=R\left[\theta_{1}, \ldots, \theta_{s}\right]$ must be a semiprime ring, as well as right and left noetherian, and of course $R\left[\theta_{1}, \ldots, \theta_{u}\right]=Q\left[\theta_{s+1}, \ldots, \theta_{u}\right]$. Now $P Q$ is a right ideal of $Q$ and $Q / P Q \cong(R / P) \otimes_{R} Q$, whence $\operatorname{pd}_{Q}(Q / P Q) \leqslant \operatorname{pd}_{R}(R / P)=n$. On the other hand, since $Q / P Q$ contains an $R$-submodule isomorphic to $R / P$, we obtain $\operatorname{pd}_{R}(Q / P Q)$ $=n$ from Lemma 14, and then Proposition 1 says that $\operatorname{pd}_{Q}(Q / P Q) \geqslant n$. Therefore $\operatorname{pd}_{Q}(Q / P Q)=n$.

Given any $j \in\{s+1, \ldots, u\}$, we must have $\delta_{j}^{*}=r_{j 1} \delta_{i}^{*}+\ldots+r_{j s} \delta_{s}^{*}$ for suitable $r_{j i} \in R$, whence $\left(\delta_{j}-r_{j 1} \delta_{1}-\ldots-r_{j s} \delta_{s}\right)(P) \subseteq P$. Setting 
$q_{j}=r_{j 1} \theta_{1}+\ldots+r_{j s} \theta_{s} \in Q$, we compute that $\left(\delta_{j}-q_{j}\right)(P Q) \subseteq P Q$. Given any $i, j \in\{s+1, \ldots, u\}$, we have

$$
\left(\delta_{i}-\sum_{k=1}^{s} r_{i k} \delta_{k}\right)(P) \subseteq P \text { and }\left(\delta_{j}-\sum_{t=1}^{s} r_{j t} \delta_{t}\right)(P) \subseteq P \text {, }
$$

from which it follows that

$$
\begin{aligned}
& {\left[\left(\delta_{j}-\sum_{t=1}^{s} r_{j t} \delta_{t}\right)\left(\delta_{i}-\sum_{k=1}^{s} r_{i k} \delta_{k}\right)\right.} \\
& \left.\quad-\left(\delta_{i}-\sum_{k=1}^{s} r_{i k} \delta_{k}\right)\left(\delta_{j}-\sum_{t=1}^{s} r_{j t} \delta_{t}\right)\right](P) \subseteq P .
\end{aligned}
$$

We compute that

$$
\begin{gathered}
\left(\delta_{j}-\sum_{t=1}^{s} r_{j t} \delta_{t}\right)\left(\delta_{i}-\sum_{k=1}^{s} r_{i k} \delta_{k}\right)-\left(\delta_{i}-\sum_{k=1}^{s} r_{i k} \delta_{k}\right)\left(\delta_{j}-\sum_{t=1}^{s} r_{j t} \delta_{t}\right) \\
=\sum_{k=1}^{s}\left[\left(\delta_{i}-\sum_{t=1}^{s} r_{i t} \delta_{t}\right)\left(r_{j k}\right)-\left(\delta_{j}-\sum_{t=1}^{s} r_{j t} \delta_{t}\right)\left(r_{i k}\right)\right] \delta_{k} ;
\end{gathered}
$$

hence we obtain

$$
\sum_{k=1}^{s}\left[\left(\delta_{i}-\sum_{t=1}^{s} r_{i t} \delta_{t}\right)\left(r_{j k}\right)-\left(\delta_{j}-\sum_{t=1}^{s} r_{j t} \delta_{t}\right)\left(r_{i k}\right)\right] \delta_{k}^{*}=0 .
$$

Inasmuch as $\delta_{1}^{*}, \ldots, \delta_{s}^{*}$ are linearly independent over $R / P$, we see that

$$
\left(\delta_{i}-\sum_{t=1}^{s} r_{i t} \delta_{t}\right)\left(r_{j k}\right)-\left(\delta_{j}-\sum_{t=1}^{s} r_{j t} \delta_{t}\right)\left(r_{i k}\right) \in P \text { for } k=1, \ldots, s,
$$

from which we compute that $\left(\delta_{i}-q_{i}\right)\left(q_{j}\right)-\left(\delta_{j}-q_{j}\right)\left(q_{i}\right) \in P Q$. According to Corollary 8 , we obtain $\mathrm{r} g l \operatorname{dim} Q\left[\theta_{s+1}, \ldots, \theta_{u}\right] \geqslant u-s+n$. Inasmuch as $u-s=\operatorname{diff} \operatorname{dim}(P)$ and $n=\operatorname{rank}(P)$, we are done.

4. Upper bounds. The purpose of this section is to introduce two kinds of upper bounds which are needed in the computation of the global dimension of $R\left[\theta_{1}, \ldots, \theta_{u}\right]$. First, we prove a theorem which shows that the global dimension of $R\left[\theta_{1}, \ldots, \theta_{u}\right]$ is the supremum of the projective dimensions of its simple modules. The second upper bound, which is needed only in the case 
that $R$ is an algebra over the rationals, shows that, for any maximal ideal $M$ of $R$, all factor modules of $R\left[\theta_{1}, \ldots, \theta_{u}\right] / M R\left[\theta_{1}, \ldots, \theta_{u}\right]$ have projective dimension at most $\operatorname{rank}(M)+\operatorname{diff} \operatorname{dim}(M)$.

For the first theorem, we need the concepts of Krull dimension (for noncommutative rings) and critical modules, as defined in [7] .

THEOREM 16. Let $R$ be any nonzero right noetherian, left coherent ring. If $\mathrm{r} g l \operatorname{dim} R=n<\infty$, then $n=\sup \left\{\operatorname{pd}_{R}(A) \mid A_{R}\right.$ is simple $\}$.

Proof. Since this is clear for $n=0$, we may assume that $n>0$. Inasmuch as $R$ is right noetherian, we have $\operatorname{GWD}(R)=n$ and $\operatorname{pd}_{R}(A)=\operatorname{wd}_{R}(A)$ for all simple modules $A_{R}$, hence it suffices to show that $R$ has a simple right module with weak dimension $n$. According to [3, Theorem 2.1], all direct products of flat right $R$-modules are flat, from which we infer that the weak dimension of any direct product of right $R$-modules equals the supremum of the weak dimensions of the factors.

In view of [7, Proposition 1.3], all finitely generated right $R$-modules have Krull dimension, and there certainly exist finitely generated right $R$-modules with weak dimension $n$. Now let $\alpha$ be minimal among the Krull dimensions of those finitely generated right $R$-modules which have weak dimension $n$, and choose some finitely generated right $R$-module $B$ such that $\mathrm{K} \operatorname{dim}(B)=\alpha$ and $\mathrm{wd}_{R}(B)=n$. Since $n>0$, we have $B \neq 0$. All factor modules of $B$ are finitely generated and hence have Krull dimension, whence [7, Theorem 2.1] says that every nonzero factor module of $B$ contains a critical submodule. Thus $B$ must have a chain of submodules $B_{0}=0<B_{1}<\ldots<B_{k}=B$ such that each $B_{i} / B_{i-1}$ is critical. Inasmuch as $\operatorname{wd}_{R}(B) \leqslant \sup \left\{\operatorname{wd}_{R}\left(B_{i} / B_{i-1}\right)\right\}$, we must have $\operatorname{wd}_{R}\left(B_{i} / B_{i-1}\right)=n$ for some $i$. Setting $A=B_{i} / B_{i-1}$, we see by [7, Lemma 1.1] that $\mathrm{K} \operatorname{dim}(A) \leqslant \alpha$, hence it follows from the minimality of $\alpha$ that $\mathrm{K} \operatorname{dim}(A)=\alpha$.

We now have a finitely generated $\alpha$-critical right $R$-module $A$ such that $\operatorname{wd}_{R}(A)=n$. We claim that $\alpha=0$, i.e., that $A$ is simple.

Assume on the contrary that $\alpha>0$. Then every nonzero submodule of $A$ is $\alpha$-critical too [7, Proposition 2.3], and thus is not simple; so $A$ has no simple submodules. Thus the intersection of all nonzero submodules of $A$ is zero, hence we obtain an embedding $A \rightarrow P$, where $P$ is the direct product of all proper factors of $A$. Since $A$ is $\alpha$-critical, each proper factor of $A$ is a finitely generated module with Krull dimension strictly less than $\alpha$, so by the minimality of $\alpha$ we see that each proper factor of $A$ has weak dimension at most $n-1$. However, this implies that $\operatorname{wd}_{R}(P) \leqslant n-1$, which contradicts Lemma 14 . Therefore $\alpha=0$ and $A$ is simple.

We now turn to considering factors of $R\left[\theta_{1}, \ldots, \theta_{u}\right] / M R\left[\theta_{1}, \ldots, \theta_{u}\right]$, 
where $M$ is a maximal ideal of $R$, and $R$ is an algebra over the rationals. For conciseness, we here use the term $u$-differential Ritt algebra to stand for a commutative $u$-differential ring which is an algebra over the rationals. In such a case, the rings $R\left[\theta_{1}, \ldots, \theta_{j}\right]$ will also be algebras over the rationals, but we do not refer to them as Ritt algebras since they are usually not commutative.

LEMMA 17. Let $R$ be any differential ring which is an algebra over the rationals, and let $M$ be any maximal right ideal of $R$. If $(\delta+a)(M) \underline{q} M$ for all $a \in R$, then $M R[\theta]$ is a maximal right ideal of $R[\theta]$.

PRoof. Suppose on the contrary that $R[\theta]$ has a right ideal $J$ such that $M R[\theta]<J<R[\theta]$, and pick an element $x \in J-M R[\theta]$ of minimal degree. Observing that $J \cap R=M$, we see that $x$ must have degree $n>0$, and we write $x=x_{0}+\ldots+x_{n} \theta^{n}$ with $x_{0}, \ldots, x_{n} \in R$ and $x_{n} \neq 0$. In view of the minimality of $n$, we infer that $x_{n} \notin M$, whence $x_{n} r+y=1$ for some $r \in R, y \in M$. Then $x r+y \theta^{n}$ has leading term $\theta^{n}$, hence $x r+y \theta^{n}$ is an element of $J-M R[\theta]$ with degree $n$. Thus, replacing $x$ by $x r+y \theta^{n}$, we may assume that $x_{n}=1$.

Given any $m \in M$, it is clear that $x m-m \theta^{n} \in J$. Observing that $x m-$ $m \theta^{n}$ has degree at most $n-1$, we obtain $x m-m \theta^{n} \in M R[\theta]$, by the minimality of $n$. Since the coefficient of $\theta^{n-1}$ in $x m-m \theta^{n}$ is $x_{n-1} m+n(\delta m)$, we thus get $x_{n-1} m+n(\delta m) \in M$. But now $\left(\delta+x_{n-1} / n\right)(M) \subseteq M$, which is impossible.

Lemma 18. Let $R$ be a u-differential Ritt algebra, and let $M$ be a maximal ideal of $R$. Assume that $s$ is a nonnegative integer such that the induced maps $\delta_{1}^{*}, \ldots, \delta_{s}^{*} \in \operatorname{Hom}_{R / M}\left(M / M^{2}, R / M\right)$ are linearly independent over $R / M$. Then $M R\left[\theta_{1}, \ldots, \theta_{s}\right]$ is a maximal right ideal of $R\left[\theta_{1}, \ldots, \theta_{s}\right]$.

Proof. We first prove the following series of statements $P_{0}, \ldots, P_{s-1}$. $P_{j}:$ If $a \in R\left[\theta_{1}, \ldots, \theta_{j}\right]$ and $r_{j+1}, \ldots, r_{s} \in R$ such that

$$
\left(a+r_{j+1} \delta_{j+1}+\ldots+r_{s} \delta_{s}\right)(M) \subseteq M R\left[\theta_{1}, \ldots, \theta_{j}\right],
$$

then $a \in R+M R\left[\theta_{1}, \ldots, \theta_{j}\right]$ and $r_{j+1}, \ldots, r_{s} \in M$.

To prove $P_{0}$, assume that we have $a, r_{1}, \ldots, r_{s} \in R$ such that $\left(a+r_{1} \delta_{1}+\ldots+r_{s} \delta_{s}\right)(M) \subseteq M$. Since $a M \subseteq M$ as well, we obtain $\left(r_{1} \delta_{1}+\ldots+r_{s} \delta_{s}\right)(M) \subseteq M$, for which it follows that $r_{1} \delta_{1}^{*}+\ldots+r_{s} \delta_{s}^{*}=0$. In view of the linear independence of $\delta_{1}^{*}, \ldots, \delta_{s}^{*}$ over $R / M$, this implies that $r_{1}, \ldots, r_{s} \in M$. Therefore $P_{0}$ holds.

Now let $0<j \leqslant s-1$ and assume that $P_{j-1}$ holds. If $P_{j}$ fails, then there exist elements $a \in R\left[\theta_{1}, \ldots, \theta_{j}\right]$ and $r_{j+1}, \ldots, r_{s} \in R$ such that 


$$
\left(a+r_{j+1} \delta_{j+1}+\ldots+r_{s} \delta_{s}\right)(M) \subseteq M R\left[\theta_{1}, \ldots, \theta_{j}\right],
$$

but either $a \notin R+M R\left[\theta_{1}, \ldots, \theta_{j}\right]$ or else some $r_{i} \notin M$. In case $a \in R+$ $\operatorname{MR}\left[\theta_{1}, \ldots, \theta_{j}\right]$, then $a M \subseteq M R\left[\theta_{1}, \ldots, \theta_{i}\right]$ and hence $\left(r_{j+1} \delta_{j+1}+\ldots+r_{s} \delta_{s}\right)(M) \subseteq M R\left[\theta_{1}, \ldots, \theta_{j}\right]$, from which we obtain $\left(r_{j+1} \delta_{j+1}+\ldots+r_{s} \delta_{s}\right)(M) \subseteq M$. In this situation, however., $P_{0}$ says that $r_{j+1}, \ldots, r_{s} \in M$, which is impossible. Thus we must have $a \notin R+$ $\operatorname{MR}\left[\theta_{1}, \ldots, \theta_{j}\right]$, and in particular $a \neq 0$. We may also assume that $a$ has the lowest degree in $\theta_{j}$ of those elements of $R\left[\theta_{1}, \ldots, \theta_{j}\right]$ for which there exist $r_{j+1}, \ldots, r_{s} \in R$ with

$$
\left(a+r_{j+1} \delta_{j+1}+\ldots+r_{s} \delta_{s}\right)(M) \subseteq M R\left[\theta_{1}, \ldots, \theta_{j}\right] .
$$

Now write $a=a_{0}+a_{1} \theta_{j}+\ldots+a_{k} \theta_{j}^{k}$, where $a_{0}, \ldots, a_{k} \in R\left[\theta_{1}, \ldots, \theta_{j-1}\right]$ and $a_{k} \neq 0 .{ }^{\bullet}$ In view of $P_{j-1}$, we must have $k>0$, and then it follows from the minimality of $k$ that $a_{k} \notin M R\left[\theta_{1}, \ldots, \theta_{j-1}\right]$.

If $k \geqslant 2$, then for any $m \in M$ we compute that

$$
\left(a+r_{j+1} \delta_{j+1}+\ldots+r_{s} \delta_{s}\right)(m)
$$

leads off with the terms $a_{k} m \theta_{j}^{k}+\left[a_{k-1} m+k a_{k}\left(\delta_{j} m\right)\right] \theta_{j}^{k-1}$, from which we obtain

$$
a_{k} m, a_{k-1} m+k a_{k}\left(\delta_{j} m\right) \in M R\left[\theta_{1}, \ldots, \theta_{j-1}\right] .
$$

First, we have $a_{k} M \subseteq M R\left[\theta_{1}, \ldots, \theta_{j-1}\right]$, hence $P_{j-1}$ says that $a_{k}=r+b$ for some $r \in R, b \in M R\left[\theta_{1}, \ldots, \theta_{j-1}\right]$. Inasmuch as $a_{k} \notin M R\left[\theta_{1}, \ldots, \theta_{j-1}\right]$, we see that $r \notin M$. Second, we have $\left(a_{k-1}+k a_{k} \delta_{j}\right)(M) \subseteq M R\left[\theta_{1}, \ldots, \theta_{j-1}\right]$, and clearly $\left(k b \delta_{j}\right)(M) \subseteq M R\left[\theta_{1}, \ldots, \theta_{j-1}\right]$ as well, whence

$$
\left(a_{k-1}+k r \delta_{j}\right)(M) \subseteq M R\left[\theta_{1}, \ldots, \theta_{j-1}\right] .
$$

According to $P_{j-1}$, we obtain $k r \in M$, and then $r \in M$ (because $R$ is a Ritt algebra). This is a contradiction.

Therefore $k<2$, so the only possibility left is $k=1$. Now $a=a_{0}+a_{1} \theta_{j}$, hence for any $m \in M$ we have

$$
\begin{aligned}
& \left(a+r_{j+1} \delta_{j+1}+\ldots+r_{s} \delta_{s}\right)(m) \\
& \quad=a_{1} m \theta_{j}+\left[a_{0} m+a_{1}\left(\delta_{j} m\right)+r_{j+1}\left(\delta_{j+1} m\right)+\ldots+r_{s}\left(\delta_{s} m\right)\right] .
\end{aligned}
$$

Thus $a_{1} M \subseteq M R\left[\theta_{1}, \ldots, \theta_{j-1}\right]$ and also 


$$
\left(a_{0}+a_{1} \delta_{j}+r_{j+1} \delta_{j+1}+\ldots+r_{s} \delta_{s}\right)(M) \subseteq M R\left[\theta_{1}, \ldots, \theta_{j-1}\right] .
$$

As above, it follows from the first inclusion that $a_{1}=r+b$ for some $r \in R-M$, $b \in M R\left[\theta_{1}, \ldots, \theta_{j-1}\right]$, and then we infer from the second inclusion that

$$
\left(a_{0}+r \delta_{j}+r_{j+1} \delta_{j+1}+\ldots+r_{s} \delta_{s}\right)(M) \subseteq M R\left[\theta_{1}, \ldots, \theta_{j-1}\right] .
$$

But now $P_{j-1}$ gives us $r \in M$, which is impossible.

Therefore $P_{j}$ must hold, and the induction works. We now return to the proof of the lemma and show that for $j=0, \ldots, s, M R\left[\theta_{1}, \ldots, \theta_{j}\right]$ is a maximal right ideal of $R\left[\theta_{1}, \ldots, \theta_{j}\right]$. For $j=0$, this is part of our hypotheses. Now let $0<j \leqslant s$ and assume that $M R\left[\theta_{1}, \ldots, \theta_{j-1}\right]$ is a maximal right ideal of $R\left[\theta_{1}, \ldots, \theta_{j-1}\right]$. In view of $P_{j-1}$, we must have

$$
\left(\delta_{j}+a\right)\left(M R\left[\theta_{1}, \ldots, \theta_{j-1}\right]\right) \underline{\Phi} M R\left[\theta_{1}, \ldots, \theta_{j-1}\right]
$$

for all $a \in R\left[\theta_{1}, \ldots, \theta_{j-1}\right]$, whence Lemma 17 shows that $M R\left[\theta_{1}, \ldots, \theta_{j}\right]$ is a maximal right ideal of $R\left[\theta_{1}, \ldots, \theta_{j}\right]$.

PROPOSITION 19. Let $R$ be a noetherian u-differential Ritt algebra, and set $T=R\left[\theta_{1}, \ldots, \theta_{u}\right]$. Let $M$ be any maximal ideal of $R$. If $J$ is any right ideal of $T$ which contains $M$, then $\operatorname{pd}_{T}(T / J) \leqslant \operatorname{rank}(M)+\operatorname{diff} \operatorname{dim}(M)$.

Proof. If $s=\operatorname{diff} \operatorname{codim}(M)$, then according to Proposition 12 the subspace $W$ of $\operatorname{Hom}_{R / M}\left(M / M^{2}, R / M\right)$ spanned by $\delta_{1}^{*}, \ldots, \delta_{u}^{*}$ has dimension $s$; hence we may arrange the indices $1, \ldots, u$ so that $\delta_{1}^{*}, \ldots, \delta_{s}^{*}$ is a basis for $W$. Setting $Q=R\left[\theta_{1}, \ldots, \theta_{s}\right]$, we now see from Lemma 18 that $M Q$ is a maximal right ideal of $Q$.

Given any $j \in\{s+1, \ldots, u\}$, we must have $\delta_{j}^{*}=r_{j 1} \delta_{1}^{*}+\ldots+r_{j s} \delta_{s}^{*}$ for suitable $r_{j i} \in R$, whence $\left(\delta_{j}-r_{j 1} \delta_{1}-\ldots-r_{j s} \delta_{s}\right)(M) \subseteq M$. Setting $q_{j}=$ $r_{j 1} \theta_{1}+\ldots+r_{j s} \theta_{s} \in Q$, we compute that $\left(\theta_{j}-q_{j}\right) M \subseteq M T$. If now $X$ denotes the set of all products of nonnegative powers of $\theta_{s+1}-q_{s+1}, \ldots, \theta_{u}-q_{u}$, then we obtain $X M T \subseteq M T$.

In particular, $X M Q \subseteq M T \subseteq J$. Observing that $T$ is generated as a right $Q$-module by $X$, we infer that $(T / J)_{Q}$ is a sum of homomorphic images of $Q / M Q$. Inasmuch as $Q / M Q$ is a simple right $Q$-module, it follows that $(T / J)_{Q}$ is isomorphic to a direct sum of copies of $Q / M Q$, whence $\operatorname{pd}_{Q}(T / J) \leqslant$ $\operatorname{pd}_{Q}(Q / M Q)$. Since $Q / M Q \cong(R / M) \otimes_{R} Q$, we also have $\operatorname{pd}_{Q}(Q / M Q) \leqslant$ $\operatorname{pd}_{R}(R / M)$. In addition, $\operatorname{pd}_{R}(R / M)=\operatorname{rank}(M)$ by Proposition 9 , and thus $\operatorname{pd}_{Q}(T / J) \leqslant \operatorname{rank}(M)$. According to Proposition $1, \operatorname{pd}_{T}(T / J) \leqslant u-s+\operatorname{rank}(M)$. Inasmuch as $u-s=\operatorname{diff} \operatorname{dim}(M)$, this gives us the required inequality. 
5. Global dimension formulas.

THEOREM 20. Let $R$ be a noetherian u-differential Ritt algebra with gl $\operatorname{dim} R<\infty$. Then

$\mathrm{r} g \mathrm{dim} R\left[\theta_{1}, \ldots, \theta_{u}\right]$

$$
=\sup \{\operatorname{rank}(P)+\operatorname{diff} \operatorname{dim}(P) \mid P \text { is a prime ideal of } R\} .
$$

Proof. If $S=R\left[\theta_{1}, \ldots, \theta_{u}\right], n=\mathrm{r} \mathrm{gl} \operatorname{dim} S$, and

$$
k=\sup \{\operatorname{rank}(P)+\operatorname{diff} \operatorname{dim}(P) \mid P \text { is a prime ideal of } R\},
$$

then $n \geqslant k$ by Proposition 15. According to Proposition $2, n \leqslant u+\mathrm{gl} \operatorname{dim} R<$ $\infty$. Inasmuch as $S$ is right and left noetherian, Theorem 16 says that there exists a simple right $S$-module $A$ with $\operatorname{pd}_{S}(A)=n$, and we note that $\operatorname{wd}_{S}(A)=n$ also.

Choose a nonzero element $x \in A$ whose $R$-annihilator $P=\{r \in R \mid x r=0\}$ is maximal among the $R$-annihilators of all nonzero elements of $A$. According to [10, Theorem 6], $P$ is a prime ideal of $R$. If $T=R_{P}\left[\theta_{1}, \ldots, \theta_{u}\right]$, then the right $R_{P}$-module $A_{P}$ can be made into a right $T$-module by defining $(a / s) \theta_{i}=$ $\left[a \theta_{i} s+a\left(\delta_{i} s\right)\right] / s^{2}$ for all $i$ and all $a / s \in A_{P}$. Since the $R$-annihilator of $x$ is $P$, the natural map $A \rightarrow A_{P}$ is not zero. However, this map is an $S$-homomorphism and $A$ is a simple $S$-module, hence $A \rightarrow A_{P}$ must be a monomorphism. In view of Lemma 14, we thus obtain $\operatorname{wd}_{S}\left(A_{P}\right)=n$.

Now $A=x S$ and thus $A_{P}=(x / 1) T$, from which we infer that $A_{P} \cong T / J$ for some right ideal $J$ of $T$ which contains $P R_{P}$. According to.Proposition 19, $\operatorname{pd}_{T}\left(A_{P}\right) \leqslant \operatorname{rank}\left(P R_{P}\right)+\operatorname{diff} \operatorname{dim}\left(P R_{P}\right)$. In view of Corollary 13, we now obtain $\operatorname{wd}_{T}\left(A_{P}\right) \leqslant \operatorname{rank}(P)+\operatorname{diff} \operatorname{dim}(P) \leqslant k$. Inasmuch as $T_{S}$ is flat by Proposition 3, $\operatorname{wd}_{S}\left(A_{P}\right) \leqslant \mathrm{wd}_{T}\left(A_{P}\right)$, and therefore $n \leqslant k$.

THEOREM 21. Let $R$ be any commutative noetherian u-differential ring such that $\mathrm{gl} \operatorname{dim} R<\infty$. Set

$$
\begin{gathered}
k=\sup \{\operatorname{rank}(P)+\operatorname{diff} \operatorname{dim}(P) \mid P \text { is a prime ideal of } R \text { and } \operatorname{char}(R / P)=0\}, \\
q=\sup \{\operatorname{rank}(M) \mid M \text { is a maximal ideal of } R \text { and } \operatorname{char}(R / M)>0\} .
\end{gathered}
$$

[In either case, if there are no ideals of the type required, the supremum is considered to be $-\infty$.] Then

$$
\mathrm{r} g \mathrm{~d} \operatorname{dim} R\left[\theta_{1}, \ldots, \theta_{u}\right]=\max \{k, q+u\} \text {. }
$$

Proof. In view of Propositions 10 and 15, we have $\mathrm{r} g l \operatorname{dim} R\left[\theta_{1}, \ldots, \theta_{u}\right]$ $\geqslant \max \{k, q+u\}$. According to Proposition 4 , the reverse inequality will hold 
provided $\mathrm{r} g l \operatorname{dim} R_{M}\left[\theta_{1}, \ldots, \theta_{u}\right] \leqslant \max \{n, q+u\}$ for each maximal ideal $M$ of $R$.

First consider the case when $\operatorname{char}(R / M)>0$. According to Proposition 9, gl $\operatorname{dim} R_{M}=\operatorname{rank}(M) \leqslant q$, hence Proposition 2 shows that $\mathrm{r} \mathrm{gl} \operatorname{dim} R_{M}\left[\theta_{1}, \ldots, \theta_{u}\right] \leqslant q+u$.

Now assume that $\operatorname{char}(R / M)=0$. Here $n R_{M} \nsubseteq M R_{M}$ for all nonzero integers $n$, hence all nonzero integers are invertible in $R_{M}$. Thus $R_{M}$ is a Ritt algebra, and so Theorem 20 is applicable. According to [10, Theorem 34], any prime ideal of $R_{M}$ must have the form $P R_{M}$ for some prime ideal $P$ of $R$ which is contained in $M$, and since $\operatorname{char}(R / M)=0$ we see that $\operatorname{char}(R / P)=0$, too. In view of Corollary 13, we obtain

$$
\operatorname{rank}\left(P R_{M}\right)+\operatorname{diff} \operatorname{dim}\left(P R_{M}\right)=\operatorname{rank}(P)+\operatorname{diff} \operatorname{dim}(P) \leqslant k,
$$

and therefore Theorem 20 shows that $\mathrm{r} g l \operatorname{dim} R_{M}\left[\theta_{1}, \ldots, \theta_{u}\right] \leqslant k$.

In particular, Theorem 21 gives a formula for the global dimension of $R[\theta]$ when $R$ is only a 1-differential ring. For this case, the formula can be improved somewhat as follows, since the differential dimension of any prime ideal $P$ depends only on whether or not $P$ is a differential ideal. Also, for this case it is possible to restrict attention to just the maximal ideals of $R$.

THEOREM 22. Let $R$ be any commutative noetherian differential ring with $\mathrm{gl} \operatorname{dim} R=n<\infty$. Let $M$ denote the collection of all differential maximal ideals of $R$, together with all maximal ideals $M$ such that $\operatorname{char}(R / M)>0$, and set $k=$ $\sup \{\operatorname{rank}(M) \mid M \in M\}$. [If $M$ is empty, then $k$ is considered to be $-\infty$.] Then $\mathrm{r} \mathrm{gl} \operatorname{dim} R[\theta]=\max \{n, k+1\}$.

Proof. According to Proposition 2, $\mathrm{r} g l \operatorname{dim} R[\theta] \geqslant n$. Inasmuch as $\operatorname{diff} \operatorname{dim}(M)=1$ for any differential maximal ideal $M$ of $R$, Theorem 21 shows that $\mathrm{r} \mathrm{gl} \operatorname{dim} R[\theta] \geqslant k+1$.

Suppose that $P$ is any prime ideal of $R$ with $\operatorname{char}(R / P)=0$. If $P$ is not maximal, then it is clear from Proposition 9 that $\operatorname{rank}(P)<n$. Since $\operatorname{diff} \operatorname{dim}(P)$ $\leqslant 1$, we get $\operatorname{rank}(P)+\operatorname{diff} \operatorname{dim}(P) \leqslant n$ in this case. Now assume that $P$ is a maximal ideal. If $P$ is not a differential ideal, then $\operatorname{diff} \operatorname{dim}(P)=0$ and $\operatorname{rank}(P)+$ $\operatorname{diff} \operatorname{dim}(P) \leqslant n$, using Proposition 9 again. On the other hand, if $P$ is a differential ideal, then $\operatorname{rank}(P)+\operatorname{diff} \operatorname{dim}(P)=1+\operatorname{rank}(P) \leqslant k+1$, by definition of $k$.

Thus we have $\operatorname{rank}(P)+\operatorname{diff} \operatorname{dim}(P) \leqslant \max \{n, k+1\}$ for all prime ideals $P$ of $R$ such that $\operatorname{char}(R / P)=0$. In view of Theorem 21, we conclude that $\mathrm{r} \mathrm{gl} \operatorname{dim} R[\theta] \leqslant \max \{n, k+1\}$.

We conclude this section by using Theorem 22 to derive a formula for the global dimension of $R[\theta]$ which involves only differential ideals of $R$. We recall 
that a proper ideal $J$ in a commutative ring $R$ is said to be primary provided all zero-divisors in the ring $R / J$ are nilpotent. .

THEOREM 23. Let $R$ be any commutative noetherian differential ring with $\mathrm{gl} \operatorname{dim} R=n<\infty$, and set $k=\sup \left\{\operatorname{pd}_{R}(R / J) \mid J\right.$ is a primary differential ideal of $R$ \}. [If $R$ has no primary differential ideals, then $k$ is considered to be $-\infty$.] Then $\mathrm{r} \mathrm{gl} \operatorname{dim} R[\theta]=\max \{n, k+1\}$.

Proof. According to Proposition 2, $\mathrm{r}$ gl $\operatorname{dim} R[\theta] \geqslant n$. Inasmuch as $R$ is semiprime by Proposition 9, Corollary 8 shows that $\mathrm{r} \operatorname{gl} \operatorname{dim} R[\theta] \geqslant k+1$.

Now consider any maximal ideal $M$ of $R$ such that $\operatorname{char}(R / M)=p>0$. If $J$ is the ideal of $R$ generated by $p R$ and $\left\{x^{p} \mid x \in M\right\}$, then as in Proposition 10 we see that $M / J$ is nilpotent and that $\operatorname{pd}_{R}(R / J)=\operatorname{pd}_{R}(R / M)$. Inasmuch as $M / J$ is nilpotent, $R / J$ must be local, from which we infer that $J$ is a primary ideal of $R$. Also, $J$ is clearly a differential ideal, whence $\operatorname{pd}_{R}(R / J) \leqslant k$. Since $\operatorname{pd}_{R}(R / M)$ $=\operatorname{rank}(M)$ by Proposition 9, we thus obtain $\operatorname{rank}(M) \leqslant k$.

Thus we have $\operatorname{rank}(M) \leqslant k$ for all maximal ideals $M$ of $R$ such that $\operatorname{char}(R / M)>0$. Since any differential maximal ideal $M$ of $R$ is a primary differential ideal, we also have $\operatorname{rank}(M) \leqslant k$ for all differential maximal ideals $M$. According to Theorem 22, we thus obtain $\mathrm{r} g l \operatorname{dim} R[\theta] \leqslant \max \{n, k+1\}$.

6. Applications. For any ring $S$ and any positive integer $u$, the Weyl algebra of degree $u$ over $S$ is the ring $A_{u}(S)=S\left[x_{1}, \ldots, x_{u}\right]\left[\theta_{1}, \ldots, \theta_{u}\right]$, where the $x_{i}$ are ordinary polynomial indeterminates, and we use the derivations $\delta_{i}=$ $\partial / \partial x_{i}$ on $S\left[x_{1}, \ldots, x_{u}\right]$. J.-E. Roos has shown that for a field $F$ of characteristic $0, \mathrm{r} \mathrm{gl} \operatorname{dim} A_{u}(F)=u[13$, Théorème 1], while G. S. Rinehart has shown that, for a field $F$ of positive characteristic, $\mathrm{r} g l \operatorname{dim} A_{u}(F)=2 u$ [11, Theorem, p. 345]. We generalize these results in the following theorem, which has also been proved (using entirely different methods) in [12, Theorem 2.6].

THEOREM 24. Let $S$ be any commutative noetherian ring with $\mathrm{gl} \operatorname{dim} S=$ $n<\infty$, and set $k=\sup \{\operatorname{rank}(M) \mid M$ is a maximal ideal of $S$ and $\operatorname{char}(S / M)>0\}$. [If $S$ has no such maximal ideals, then $k$ is considered to be $-\infty$.] Then for any positive integer $u, \mathrm{r} \mathrm{gl} \operatorname{dim} A_{u}(S)=\max \{n+u, k+2 u\}$.

Proof. Set $R=S\left[x_{1}, \ldots, x_{u}\right]$ and $\delta_{i}=\partial / \partial x_{i}$ for $i=1, \ldots, u$. Since $\mathrm{gl} \operatorname{dim} R=n+u$, Proposition 2 shows that $\mathrm{r} \mathrm{gl} \operatorname{dim} A_{u}(S) \geqslant n+u$.

If $S$ has any maximal ideals $M$ such that $\operatorname{char}(S / M)>0$, then we may choose such an $M$ with $\operatorname{rank}(M)=k$. Inasmuch as $S / M$ is a field, the ring $R / M R$ $\cong(S / M)\left[x_{1}, \ldots, x_{u}\right]$ has Krull dimension $u$, whence $R / M R$ must have a maximal ideal $K / M R$ of rank $u$. Then $K$ is a maximal ideal of $R$ such that $\operatorname{char}(R / K)>0$, and clearly $\operatorname{rank}(K) \geqslant k+u$, hence Theorem 21 says that $\mathrm{r} g l \operatorname{dim} A_{u}(S) \geqslant$ $k+2 u$. 
Therefore $\mathrm{r} g l \operatorname{dim} A_{u}(S) \geqslant \max \{n+u, k+2 u\}$. According to Theorem 21, to prove the reverse inequality it is enough to show that $\operatorname{rank}(M) \leqslant k+u$ for any maximal ideal $M$ of $R$ with $\operatorname{char}(R / M)>0$, and that $\operatorname{rank}(P)+\operatorname{diff} \operatorname{dim}(P)$ $\leqslant n+u$ for any prime ideal $P$ of $R$ such that $\operatorname{char}(R / P)=0$.

First consider any maximal ideal $M$ of $R$ for which $\operatorname{char}(R / M)>0$. Choosing a maximal ideal $K$ of $S$ which contains $S \cap M$, we have $\operatorname{char}(S / K)>0$ and so $\operatorname{rank}(S \cap M) \leqslant \operatorname{rank}(K) \leqslant k$. By induction on [10, Theorem 149], we find that $\operatorname{rank}(M) \leqslant k+u$.

Now consider any prime ideal $P$ of $R$ with $\operatorname{char}(R / P)=0$, and set $s=$ $\operatorname{diff} \operatorname{dim}(P)$. If $T=R_{P}, M=P R_{P}$, and $W$ is the subspace of $\operatorname{Hom}_{T / M}\left(M / M^{2}, T / M\right)$ spanned by $\delta_{1}^{*}, \ldots, \delta_{u}^{*}$, then by Proposition $12 W$ has dimension $u-s$. Thus we may arrange the indices $1, \ldots, u$ so that $\delta_{s+1}^{*}, \ldots, \delta_{u}^{*}$ is a basis for $W$. Set $Q=P \cap\left(S\left[x_{1}, \ldots, x_{s}\right]\right)$ and note that $S\left[x_{1}, \ldots, x_{s}\right] / Q$ has characteristic 0 . We claim that $\delta_{i}(Q) \subseteq Q$ for $i=1, \ldots$, s. Given $1 \leqslant i \leqslant s$, we must have $\delta_{i}^{*}=t_{s+1} \delta_{s+1}^{*}+\ldots+t_{u} \delta_{u}^{*}$ for suitable $t_{j} \in T$. Multiplying out the denominators in this equation, we obtain

$$
a \delta_{i}^{*}=r_{s+1} \delta_{s+1}^{*}+\ldots+r_{u} \delta_{u}^{*} \text { for some } a \in R-P, r_{s+1}, \ldots, r_{u} \in R \text {. }
$$

Thus $\left(a \delta_{i}-r_{s+1} \delta_{s+1}-\ldots-r_{u} \delta_{u}\right)(M) \subseteq M$, from which we infer that

$$
\left(a \delta_{i}-r_{s+1} \delta_{s+1}-\ldots-r_{u} \delta_{u}\right)(P) \subseteq P .
$$

Since $Q \subseteq P$ and $\delta_{s+1}, \ldots, \delta_{u}$ all vanish on $Q$, we thus obtain $a \delta_{i}(Q) \subseteq P$. Now $P$ is a prime ideal of $R$ and $a \in R-P$, hence it follows that $\delta_{i}(Q) \subseteq P$, from which we conclude that $\delta_{i}(Q) \subseteq Q$, as claimed.

All of the rings $S\left[x_{1}, \ldots, x_{i}\right] /\left(Q \cap S\left[x_{1}, \ldots, x_{i}\right]\right) \quad(i=1, \ldots, s)$ have characteristic 0 , hence with the help of the relations $\delta_{i}(Q) \subseteq Q$ an easy induction shows that $Q \cap\left(S\left[x_{1}, \ldots, x_{i}\right]\right)=(Q \cap S)\left[x_{1}, \ldots, x_{i}\right]$ for each $i=1, \ldots, s$. Consequently $Q=(Q \cap S)\left[x_{1}, \ldots, x_{s}\right]$, whence [10, Theorem 149] shows that $\operatorname{rank}(Q)=\operatorname{rank}(Q \cap S)$. That same theorem also shows that $\operatorname{rank}(P) \leqslant u-s+$ $\operatorname{rank}(Q)$, and it is clear from Proposition 12 that $\operatorname{rank}(Q \cap S) \leqslant n$, hence we obtain $\operatorname{rank}(P) \leqslant n+u-s$. Therefore $\operatorname{rank}(P)+\operatorname{diff} \operatorname{dim}(P) \leqslant n+u$.

COROLlaRY 25. Let $S$ be any commutative noetherian ring with $\mathrm{gl} \operatorname{dim} S=$ $n<\infty$, and let $u$ be any positive integer. If $S$ is an algebra over the rationals, then $\mathrm{r} g l \operatorname{dim} A_{u}(S)=n+u$.

Corollary 25 has also been obtained in [1, Corollary 2.6] .

Given any ring $S$ and any positive integer $u$, then following [2] we can define a ring $F_{u}(S)=S\left[\left[x_{1}, \ldots, x_{u}\right]\right]\left[\theta_{1}, \ldots, \theta_{u}\right]$ analogous to the Weyl algebra $A_{u}(S)$. If $S$ is a commutative noetherian ring with $\operatorname{gl} \operatorname{dim} S=n<\infty$, and if $S$ is an algebra over the rationals, then J.-E. Björk has shown in [2, The- 
orem 4.2] that $\mathrm{r} g l \operatorname{dim} F_{u}(S)=n+u$. We shall generalize this result, but first some facts about power series rings must be developed. [We note that our proofs do not depend on Björk's result, and our methods are completely different from his.]

LEMma 26. Let $S$ be a commutative noetherian ring with $\mathrm{gl} \operatorname{dim} S=n<$ $\infty$. If $u$ is any positive integer, then $g l \operatorname{dim} S\left[\left[x_{1}, \ldots, x_{u}\right]\right]=n+u$.

Proof. It obviously suffices to prove the case $n=1$. The indeterminate $x$ lies in the Jacobson radical of $S[[x]]$, and $x$ is not a zero-divisor in $S[[x]]$. Since $S[[x]] / x S[[x]] \cong S,[9$, Part III, Theorem 10] shows that $\mathrm{r}$ gl dim $S[[x]]$ $=n+1$.

LEMMA 27. If $F$ is a field and $u$ any positive integer, then $F\left[\left[x_{1}, \ldots, x_{u}\right]\right]$ has Krull dimension $u$.

Proof. This is immediate from Lemma 26 and Proposition 9.

For use in the next lemma, we recall that if $S$ is a commutative local ring with maximal ideal $M$, then $S[[x]]$ is a local ring with maximal ideal generated by $M$ and $x$. Clearly, the ideal $J$ of $S[[x]]$ generated by $M$ and $x$ is a maximal ideal. Also, if $p$ is any element of $S[[[x]]$ which does not belong to $J$, then the constant term of $p$ is not in $M$ and so is invertible in $S$, whence $p$ is invertible in $S[[x]]$.

Lemma 28. Let $S$ be a commutative noetherian ring, and let $Q$ be any prime ideal of $S, u$ any positive integer. Then $Q\left[\left[x_{1}, \ldots, x_{u}\right]\right]$ is a prime ideal of $S\left[\left[x_{1}, \ldots, x_{u}\right]\right]$ with rank equal to $\operatorname{rank}(Q)$. Also, if $P$ is any prime ideal of $S\left[\left[x_{1}, \ldots, x_{u}\right]\right]$ such that $P \cap S=Q$, then $\operatorname{rank}(P) \leqslant u+\operatorname{rank}(Q)$.

Proof. We may obviously assume that $\operatorname{rank}(Q)<\infty$. Also, we clearly need only prove the case $u=1$. Finally, since $P$ is disjoint from $S-Q$, all the ranks we are interested in remain the same after localizing at $Q$, hence we may assume, without loss of generality, that $S$ is local with maximal ideal $Q$. As remarked above, it follows that $S[[x]]$ is local with maximal ideal $M$ generated by $Q$ and $x$.

Now $M$ is a prime ideal in the noetherian ring $S[[x]]$, and $x$ is an element of $M$ which is not a zero-divisor in $S[[x]]$, hence [10, Theorem 155] says that the $\operatorname{rank}$ of $M / x S[[x]]$ in $S[[x]] / x S[[x]]$ equals $\operatorname{rank}(M)-1$. Inasmuch as $S[[x]] / x S[[x]] \cong S$, we infer that $\operatorname{rank}(M / x S[[x]])=\operatorname{rank}(Q)$, and thus $\operatorname{rank}(M)=1+\operatorname{rank}(Q)$. Observing that $P \subseteq M$, we obtain $\operatorname{rank}(P) \leqslant 1+\operatorname{rank}(Q)$. Finally, since $\operatorname{rank}(M)=1+\operatorname{rank}(Q)<\infty$ and $Q[[x]]$ is properly contained in $M$, we must have $\operatorname{rank}(Q[[x]]) \leqslant \operatorname{rank}(Q)$, from which we conclude that $\operatorname{rank}(Q[[x]])=\operatorname{rank}(Q)$. 
With the help of these three lemmas, we may use the proof of Theorem 24, mutatis mutandis, to prove the following generalization of Björk's theorem:

THEOREM 29. Let $S$ be any commutative noetherian ring with $\mathrm{gl} \operatorname{dim} S=$ $n<\infty$, and set $k=\sup \{\operatorname{rank}(M) \mid M$ is a maximal ideal of $S$ and $\operatorname{char}(S / M)>0\}$. [If $S$ has no such maximal ideals, then $k$ is considered to be $-\infty$.] Then, for any positive integer $u$,

$$
\mathrm{r} g l \operatorname{dim} F_{u}(S)=\max \{n+u, k+2 u\}
$$

J. Cozzens and J. Johnson have shown that for any $u$-differential field $F$, $\mathrm{r} g l \operatorname{dim} F\left[\theta_{1}, \ldots, \theta_{u}\right]=u \quad[4$, Theorem $1(\mathrm{~b})]$. In view of Corollary 8 and Proposition 2 , this result generalizes to semisimple artinian rings:

THEOREM 30. If $R$ is any semisimple artinian $u$-differential ring, then $\mathrm{r} g \mathrm{dim} R\left[\theta_{1}, \ldots, \theta_{u}\right]=u$.

\section{REFERENCES}

1. S. M. Bhatwadekar, On the global dimension of Ore-extensions, Nagoya Math. J. 50 (1973), 217-225. MR 47 \#8515.

2. J.-E. Björk, The global homological dimension of some algebras of differential operators, Invent. Math. 17 (1972), 67-78. MR 47 \#8619.

3. S. U. Chase, Direct products of modules, Trans. Amer. Math. Soc. 97 (1960), 457473. MR 22 \#11017.

4. J. Cozzens and J. Johnson, Some applications of differential algebra to ring theory, Proc. Amer. Math. Soc. 31 (1972), 354-356. MR 44 \#6662.

5. K. R. Goodearl, Global dimension of differential operator rings, Proc. Amer. Math. Soc. 45 (1974), 315-322.

6. N. S. Gopalakrishnan and R. Sridharan, Homological dimension of Ore-extensions, Pacific J. Math. 19 (1966), 67-75. MR 34 \#223. (1973).

7. R. Gordon and J. C. Robson, Krull dimension, Mem. Amer. Math. Soc. No. 133

8. I. N. Herstein, Noncommutative rings, Carus Math. Monographs, no. 15, Math. Assoc. Amer.; distributed by Wiley, New York, 1968. MR 37 \#2790. $\# 4345$.

9. I. Kaplansky, Fields and rings, Univ. of Chicago Press, Chicago, Ill., 1969. MR 42

10. - Commutative rings, Allyn and Bacon, Boston, Mass., 1970. MR 40 \#7234.

11. G. S. Rinehart, Note on the global dimension of a certain ring, Proc. Amer. Math. Soc. 13 (1962), 341-346. MR 25 \#1196.

12. G. S. Rinehart and A. Rosenberg, The global dimension of Ore extensions and Weyl algebras, A Collection of Papers in Honor of Samuel Eilenberg, Academic Press, New York, 1975.

13. J.-E. Roos, Détermination de la dimension homologique globale des algèbres de Weyl, C. R. Acad. Sci. Paris Sér. A-B 274 (1972), A23-A26. MR 45 \#1995.

14. F. L. Sandomierski, Semisimple maximal quotient rings, Trans. Amer. Math. Soc. 128 (1967), 112-120. MR 35 \#5473.

DEPARTMENT OF MATHEMATICS, UNIVERSITY OF UTAH, SALT LAKE CITY, UTAH 84112 